\title{
Objective Definition of Monofloral and Polyfloral Honeys Based on NMR
}

Metabolomic Profiling

Elisabetta Schievano ${ }^{1 *}$, Claudia Finotello ${ }^{1}$, Jalal Uddin ${ }^{1}$, Stefano Mammi ${ }^{1}$, Lucia Piana $^{2}$

${ }^{1}$ Department of Chemical Sciences, Università di Padova, via Marzolo 1, 35131 Padova, Italy

${ }^{2}$ Piana Ricerca e Consulenza s.r.l. a socio unico, Via dei Mille 39, 40024 Castel San Pietro Terme (BO), Italy

*Correspondence: elisabetta.schievano@unipd.it Phone: +39 0498275742. Fax +390498275829 
Table 1S. Chemical structures and ${ }^{1} \mathrm{H}$ proton resonances in $\mathrm{CDCl}_{3}$ of asphodel (1) and thistle $(\mathbf{2}, \mathbf{3})$ honey markers.

\begin{tabular}{|l|l|}
\hline 1-Methylsiringate & $\begin{array}{l}7.34(\mathrm{~s}, 2 \mathrm{H}, \mathbf{2 , 6}) ; \\
3.91(\mathrm{~s}, 3 \mathrm{H}, \mathbf{1 0}) ; \\
3.96(\mathrm{~s}, 6 \mathrm{H}, \mathbf{7 , 8}) ; \\
5.90(\mathrm{~s} \mathrm{br}, 1 \mathrm{H}, \mathbf{9})\end{array}$ \\
\hline
\end{tabular}

Table 2S. OPLS-DA models for the one- $v s$-all strategy and PLS-DA one- $v s$-one

\begin{tabular}{|c|c|c|c|}
\hline \multicolumn{5}{|c|}{ OPLS-DA (one-versus-one) } \\
\hline $\mathbf{R}^{\mathbf{2}} \mathbf{X}$ (cum) & $\mathbf{R}^{\mathbf{2}} \mathbf{Y}$ (cum) & $\mathbf{Q}^{\mathbf{2}}$ (cum) & Botanical origin \\
\hline 0.387 & 0.875 & 0.856 & Citrus (CI) \\
\hline 0.451 & 0.741 & 0.685 & Acacia (AC) \\
\hline 0.367 & 0.873 & 0.852 & Chestnut (CH) \\
\hline 0.393 & 0.838 & 0.821 & Linden (LI) \\
\hline 0.407 & 0.816 & 0.789 & Eucalyptus (EU) \\
\hline 0.48 & 0.508 & 0.378 & Wildflower (WF) \\
\hline 0.487 & 0.692 & 0.633 & Honeydew (HD) \\
\hline 0.375 & 0.321 & 0.241 & Sulla (SU) \\
\hline 0.487 & 0.652 & 0.565 & Rhododendron (RH) \\
\hline 0.328 & 0.773 & 0.737 & Thistle (TH) \\
\hline 0.589 & 0.826 & 0.751 & Sunflower (SF) \\
\hline 0.221 & 0.819 & 0.812 & Asphodel (AS) \\
\hline 0.282 & 0.544 & 0.472 & Apple (AP) \\
\hline 0.482 & 0.717 & 0.630 & Ailanthus (AI) \\
\hline 0.471 & 0.908 & 0.867 & Cherry (CY) \\
\hline 0.405 & 0.76 & 0.727 & Alfalfa (AA) \\
\hline \multicolumn{4}{|c|}{ PLS-DA(one- versus-one) } \\
\hline 0.629 & 0.915 & 0.802 & AC versus SU \\
\hline 0.452 & 0.843 & 0.768 & AI versus LI \\
\hline 0.599 & 0.896 & 0.85 & CI versus SU \\
\hline 0.472 & 0.871 & 0.78 & AC versus RH \\
\hline 0.787 & 0.942 & 0.92 & AS versus CI \\
\hline
\end{tabular}


Table 3S. Class assignment of the test set (272 samples) based on NMR approach. In the fourth column the assigned class and the principal floral contributions (in brackets) are reported.

\begin{tabular}{|c|c|c|c|c|}
\hline $\begin{array}{l}\text { Botanical } \\
\text { origin } \\
\text { declared }\end{array}$ & $\begin{array}{l}\text { N. of } \\
\text { samples }\end{array}$ & $\begin{array}{l}\text { Compliant } \\
\text { samples }\end{array}$ & $\begin{array}{l}\text { Non-compliant } \\
\text { samples }\end{array}$ & Sensitivity (\%) \\
\hline Citrus (CI) & 48 & 45 & $\begin{array}{l}1 \mathrm{PO}(\mathrm{CI}, \mathrm{AS}, \mathrm{TH}) \\
1 \mathrm{PO}(\mathrm{CI}, \mathrm{AS}, \mathrm{HD}) \\
1 \mathrm{PO}(\mathrm{CI}, \mathrm{EU}, \mathrm{HD})\end{array}$ & 98 \\
\hline Acacia (AC) & 30 & $26+2^{a}$ & $2 \mathrm{PO}(\mathrm{AC}, \mathrm{WF}, \mathrm{HD})$ & $87(93)^{a}$ \\
\hline Chestnut $(\mathrm{CH})$ & 25 & 25 & & 100 \\
\hline Linden (LI) & 25 & 25 & & 100 \\
\hline Eucalyptus (EU) & 26 & 24 & $\begin{array}{l}1 \mathrm{PO}(\mathrm{EU}, \mathrm{AC}, \mathrm{WF}) \\
1 \mathrm{CI}\end{array}$ & 92 \\
\hline Wildflower (WF) & 18 & 12 & $5 \mathrm{PO}$ & 67 \\
\hline Honeydew (HD) & 15 & 13 & $\begin{array}{l}1 \mathrm{PO}(\mathrm{HD}, \mathrm{AC}, \mathrm{WF}) \\
1 \mathrm{PO}(\mathrm{HD}, \mathrm{EU}, \mathrm{SU})\end{array}$ & 87 \\
\hline Sulla (SU) & 18 & $4+13^{a}$ & $1 \mathrm{CI}^{b}$ & $22(94)^{a}$ \\
\hline Rhododendron (RH) & 10 & $8+1^{c}$ & $1 \mathrm{PO}(\mathrm{RH}, \mathrm{AC}, \mathrm{HD})$ & $80(90)^{c}$ \\
\hline Thistle (TH) & 12 & 10 & $\begin{array}{l}1 \mathrm{PO}(\mathrm{TH}, \mathrm{AS}, \mathrm{EU}) \\
1 \mathrm{PO}(\mathrm{AS}, \mathrm{TH}, \mathrm{EU})\end{array}$ & 83 \\
\hline Sunflower (SF) & 10 & 9 & $1 \mathrm{PO}(\mathrm{SF}, \mathrm{WF}, \mathrm{CI})$ & 90 \\
\hline Asphodel (AS) & 14 & 13 & $1 \mathrm{CI}^{d}$ & 93 \\
\hline Ailanthus (AI) & 15 & 8 & $\begin{array}{l}4 \mathrm{LI}^{e} \\
1 \mathrm{PO}(\mathrm{AI}, \mathrm{HD}) \\
2 \mathrm{PO}(\mathrm{AI}, \mathrm{LI}, \mathrm{AC})\end{array}$ & 53 \\
\hline Cherry (CY) & 6 & 6 & & 100 \\
\hline Alfalfa (AA) & 0 & & & \\
\hline Apple (AP) & 0 & & & \\
\hline
\end{tabular}

${ }^{a}$ Class defined by a SU-versus-AC PLS-DA model.

${ }^{b}$ Class defined by a SU-versus-CI PLS-DA model.

${ }^{c}$ Class defined by a RH-versus-AC PLS-DA model.

${ }^{d}$ Class defined by a AS-versus-CI PLS-DA model.

${ }^{e}$ Class defined by a AI-versus-LI PLS-DA model. 